\title{
Laboratory Studies of Ion Chemistry in the Interstellar Medium
}

\author{
V. M. Bierbaum ${ }^{1}$ \\ ${ }^{1}$ Department of Chemistry and Biochemistry \& Center for Astrophysics and Space Astronomy, \\ University of Colorado, Boulder, Colorado 80309-0215, USA \\ email: veronica.bierbaum@colorado.edu
}

\begin{abstract}
Studies of gas phase ion-neutral reactions provide insight into many areas of astrochemistry, including the elusive characterization of the Diffuse Interstellar Bands (DIBs). This presentation gives an overview of our experimental studies of several classes of positive and negative ions, using the flowing afterglow-selected ion flow tube and a newly modified ion trap. Earlier studies of carbon chain anions and polycyclic aromatic hydrocarbon (PAH) cations, both of which have been suggested as carriers of the DIBs, are described. More recent work including isomeric PAHs, nitrogen-containing PAHs, negative ions of PAHs, and negative ions of 5 -membered heterocyclic rings are discussed. Finally, the study of quantitative thermochemistry by coupling our results with data from Photoelectron Spectroscopy is described.
\end{abstract}

Keywords. Astrochemistry, methods: laboratory, molecular processes, ISM: general, ISM: molecules, ISM: atoms, astrobiology

\section{Introduction}

A full understanding of astrophysical environments, and ultimately of the Diffuse Interstellar Bands (DIBs), requires the interplay of astronomical observations, experiments in chemistry and spectroscopy, theoretical calculations, and comprehensive modeling. Our research has focused on experimental studies of the reactions of gas phase ions to provide kinetic, thermodynamic, and mechanistic information. Despite their relatively low abundance, ions can be important in many environments since the absence of reaction barriers often allows the processes to be fast, even at low temperatures. We employ two techniques, a flowing afterglow-selected ion flow tube and a modified commercial ion trap, for these studies. Development of several different ion sources enables access to a wide array of gaseous ionic species. This presentation will first describe the instrumentation and then provide an overview of our results.

\section{Experimental}

Our laboratory studies utilize the flowing afterglow-selected ion flow tube (FA-SIFT, Fig. 1), which has been described previously (Van Doren et al. 1987; Snow \& Bierbaum 2008); the four regions allow ion production, selection, reaction, and detection. Ions are generated in a flow tube source by a variety of techniques, including electron ionization of a precursor gas. The desired reactant ion is mass-selected with a quadrupole mass filter and injected into the reaction flow tube, entrained in a flow of helium buffer gas, and detected. Thus a single type of ion can be selected and studied in the absence of all other ions and precursors. The neutral reagents are added through a manifold of inlets, and the ion-neutral reaction occurs throughout the remainder of the flow tube. By monitoring the reactant and product ion intensities as a function of reaction distance, which is proportional to reaction time, rate constants and product distribution are readily 


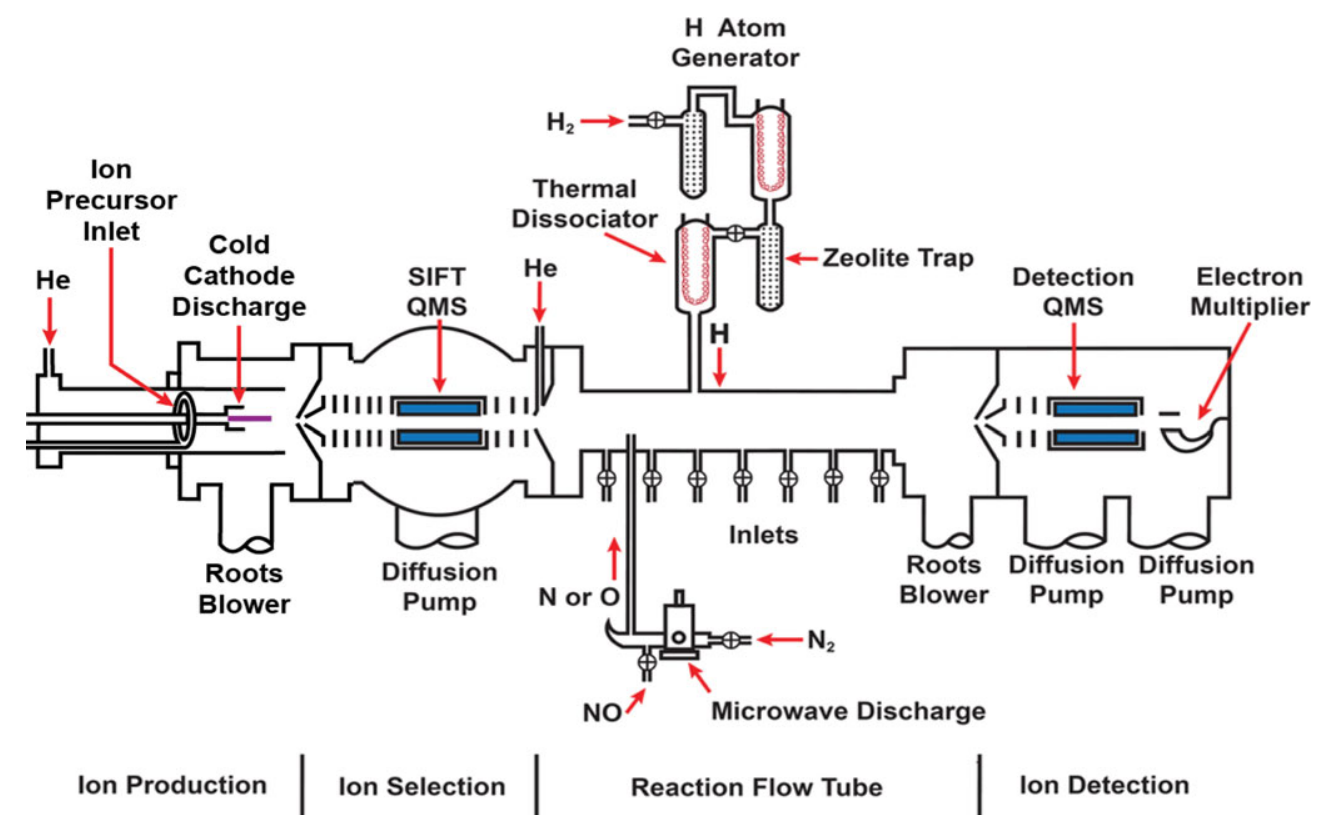

Figure 1. The Flowing Afterglow-Selected Ion Flow Tube (FA-SIFT).

determined. Alternatively, the flow of the neutral reagent can be varied to obtain kinetics. In addition to stable molecular reactants, atomic reactants can be generated and studied. $\mathrm{H}$-atom is formed by thermal dissociation of $\mathrm{H}_{2}, \mathrm{~N}$-atom is formed by microwave discharge of $\mathrm{N}_{2}$, and $\mathrm{O}$-atom is produced by titration of $\mathrm{N}$-atom with $\mathrm{NO}$. The technique has several valuable features for the study of ion chemistry. In particular, the exceptional chemical versatility, provided by a variety of ion and neutral sources, is important for generating and quantitatively studying a wide array of species relevant to interstellar chemistry. These sources include discharge techniques, Penning Ionization, Electrospray Ionization, and Laser Induced Acoustic Desorption.

We have recently acquired a rebuilt Thermo Finnigan LCQ Deca XP Plus Ion Trap, and introduced modifications that allow the study of ion-molecule chemistry. Neutral reagents are introduced into the helium flow using a syringe pump, creating pressures of $10^{-5}$ and $10^{-3}$ Torr, respectively, of neutral reactant and helium in the trap. Reaction kinetics are monitored by varying the time that ions are trapped in the presence of the neutral reactants; rate constants between $10^{-13}$ and $10^{-9} \mathrm{~cm}^{3}$ molecule ${ }^{-1} \mathrm{~s}^{-1}$ can be determined. Our semi-logarithmic plots of ion counts versus reaction time are linear, and rate constants are in agreement with literature values, confirming the success of our modified instrument in measuring accurate kinetic data. The Electrospray Ionization source provides a wide array of reactant cations and anions, including those of biomolecules and PAHs.

\section{Results}

Over the years, we have characterized the reactions of many classes of positive and negative ions. This presentation will describe our earlier studies of carbon chain anions and then of polycyclic aromatic hydrocarbon (PAH) cations, both of which have been suggested as carriers of the DIBs. More recent work including isomeric PAHs, nitrogencontaining PAHs, negative ions of PAHs, and negative ions of 5-membered heterocyclic 


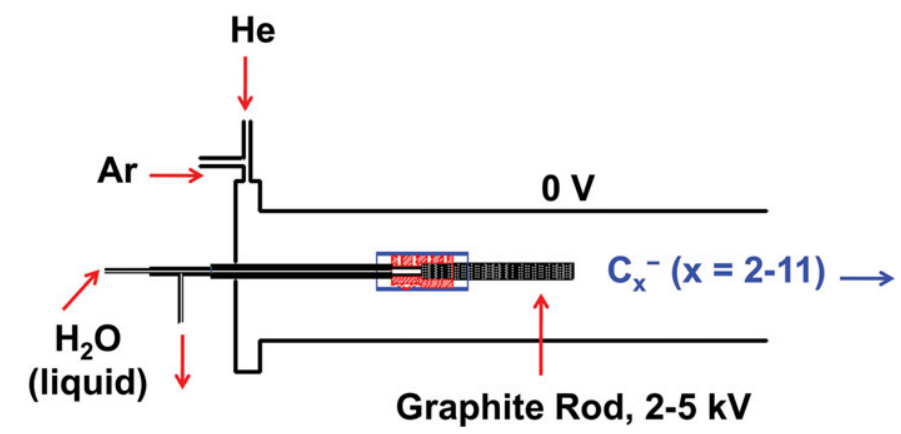

Figure 2. Generation of carbon-chain anions using a graphite rod discharge.

rings will be discussed. Finally, the study of quantitative thermochemistry by coupling the results of the FA-SIFT with data from Photoelectron Spectroscopy will be described.

Carbon chain anions. In response to the suggestion that $\mathrm{C}_{7}^{-}$could be a DIB carrier (Tulej et al. 1998), we generated and studied pure carbon chain anions, $\mathrm{C}_{x}^{-}$. These ions with $\mathrm{x}=1-11$ can be formed using a DC discharge as shown in Fig. 2; a graphite rod is raised to several kilovolts relative to the flow tube. An anion with a specific mass-tocharge ratio is selected with the SIFT quadrupole, and injected into the reaction flow tube for study. None of the carbon chain anions react with molecular hydrogen, but they react efficiently with hydrogen atoms by associative detachment; the larger carbanions also show simple addition to form the hydrogenated carbon chain anions (Barckholtz et al. 2001).

Reactions of the carbon chain anions with atomic oxygen occur rapidly by associative detachment and by the loss of a single carbon atom to form carbon monoxide and an anion with one fewer carbon atom. Reactions of carbon chain anions with nitrogen atoms are somewhat slower, but show a rich variety of pathways that involve formation of carbonnitrogen bonds (Eichelberger et al. 2007; Yang et al. 2010).

Nitrogen-containing carbanions, $\mathrm{C}_{x} \mathrm{~N}_{y}^{-}$, can be generated with the graphite rod discharge in the presence of molecular nitrogen (Yang et al. 2011). Ions containing one or two nitrogen atoms undergo rapid associative detachment with a hydrogen atom; in contrast the $\mathrm{C}_{2} \mathrm{~N}_{3}^{-}$and $\mathrm{C}_{4} \mathrm{~N}_{3}^{-}$ions show no measurable reactivity. Our computational studies indicate that there are high energy transition states along the reaction surface, and only endothermic channels exist. Thus, these ions will exhibit high stability in the ISM, even in regions of high $\mathrm{H}$-atom density.

Polycyclic Aromatic Hydrocarbon Cations. The neutral and ionic forms of the polycyclic aromatic hydrocarbons are likely the carriers of the unidentified infrared emissions; moreover, PAHs have been proposed as carriers of the DIBs (Joblin \& Tielens 2011). Thus we have undertaken a comprehensive study of their ion chemistry (Le Page et al. 2001). The PAH cations can be formed by electron ionization of the parent neutrals; however, these high-energy collisions often result in ring opening and fragmentation processes. We have found that Penning Ionization with electronically excited metastable argon atoms, illustrated in Fig. 3, provides less energetic ionization, resulting in intact molecular cations of PAHs. Using this approach, we have studied the reactions of the positive ions of benzene, naphthalene, pyrene, and coronene with several molecular and atomic reagents. We have found that reactivity with $\mathrm{H}$-atom and $\mathrm{O}$-atom are moderately rapid, and essentially independent of PAH size. In contrast, the reactivity of PAH cations with $\mathrm{N}$-atoms decreases rapidly as the PAH size increases. 


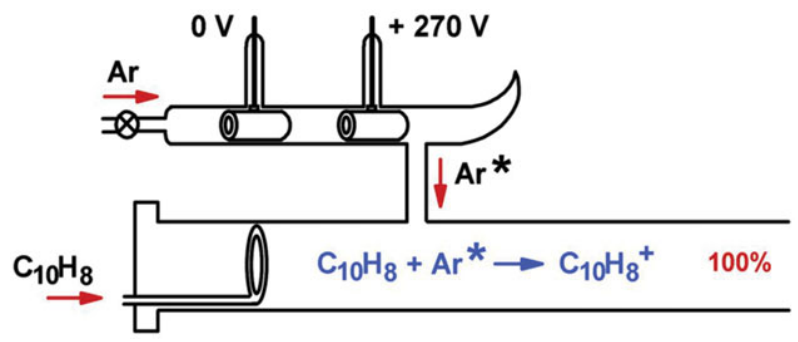

Figure 3. Formation of parent molecular cations of PAHs using Penning ionization by metastable argon atoms.

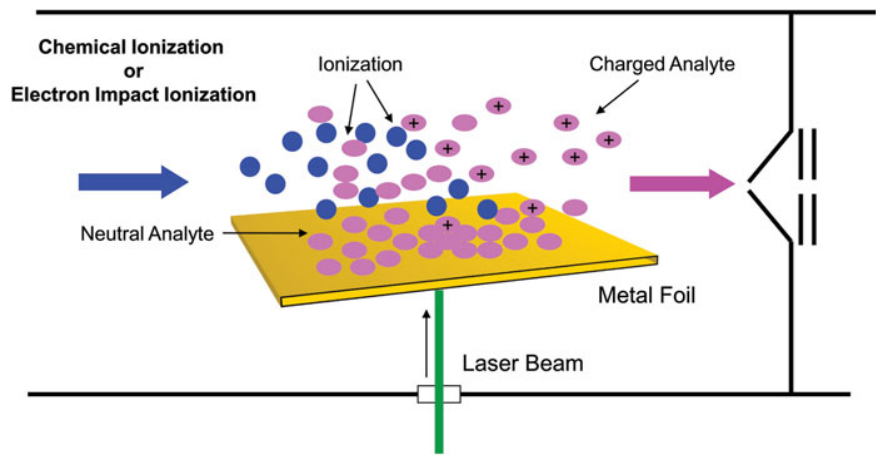

Figure 4. Schematic of laser-induced acoustic desorption.

Isomeric PAHs and Nitrogen-containing PAHs. Our studies of the chemistry of PAH ions are limited by the low volatility of the parent molecules. We have therefore implemented a new approach, Laser-Induced Acoustic Desorption, which has been extensively developed by Hilkka Kenttämaa and her research group (Shea et al. 2007). With this method, PAHs are uniformly deposited onto a thin titanium foil and mounted in the ion source region of the instrument. Pulses of $532 \mathrm{~nm}$ laser light impinge on the back of the foil as shown in Fig. 4; an acoustic wave is induced, and the intact analyte molecules are desorbed from the surface. The neutral species can be directly studied; however, in our experiments, various techniques are utilized to generate the parent PAH ions. We have recently studied the (1) naphthalene cation $\left(\mathrm{C}_{10} \mathrm{H}_{8}^{+}\right)$and the pair of isomeric nitrogen-containing analogs quinoline and isoquinoline $\left(\mathrm{C}_{9} \mathrm{H}_{7} \mathrm{~N}^{+}\right)$; (2) the anthracene cation $\left(\mathrm{C}_{14} \mathrm{H}_{10}^{+}\right)$and the nitrogen containing analog acridine $\left(\mathrm{C}_{13} \mathrm{H}_{9} \mathrm{~N}^{+}\right)$; (3) the phenanthrene cation $\left(\mathrm{C}_{14} \mathrm{H}_{10}^{+}\right)$, an isomer of anthracene, and the pair of isomeric nitrogencontaining analogs phenanthridine and benzo[h]quinoline $\left(\mathrm{C}_{13} \mathrm{H}_{9} \mathrm{~N}^{+}\right)$; and (4) the tetracene cation $\left(\mathrm{C}_{18} \mathrm{H}_{12}^{+}\right)$, which was generated using LIAD. Preliminary results indicate that the incorporation of nitrogen does not affect the reactivity of the cation with H-atom; however, changing from a "linear" to "angular" PAH cation shows a small but reproducible increase in reactivity. These results are currently being prepared for publication.

Negative Ions of PAHs. Negative ions formed by the deprotonation of PAHs have moderate electron binding energies, and are expected to exist in several regions of the interstellar medium. We have found that these anions are strong bases and readily deprotonate most molecules including methanol, acetone, acetaldehyde, acetonitrile, and acetylene. Phenide, the prototypical aromatic anion, reacts with carbon dioxide by addition to form 


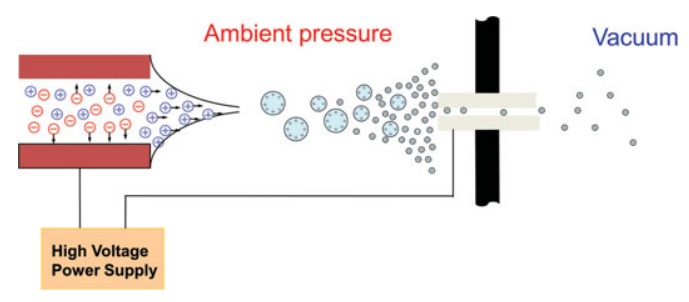

Figure 5. Schematic of Electrospray Ionization.

the carboxylate ion; reaction with nitrous oxide proceeds both by addition and by $\mathrm{O}$-atom abstraction to form phenoxide anion; reaction with alkyl halides occurs by nucleophilic substitution, but elimination reactions, for example with diethyl ether, do not readily occur. Reaction with $\mathrm{H}$-atom proceeds by facile associative detachment. These results have been recently published (Demarais et al. 2012).

Negative Ions of 5-membered Heterocyclic Rings. Five-membered rings containing three carbon atoms, a nitrogen atom, and either an oxygen or sulfur atom, have astrobiological relevance as precursors of nucleobases. However, their chemistry and thermochemistry are completely uncharacterized. We have utilized electrospray ionization (Fenn et al. 1990), illustrated in Fig. 5, in our newly modified ion trap to generate the deprotonated carboxylate ions of oxazole, thiazole, and isothiazole. Collision induced dissociation then removes the carbon dioxide moiety to produce the carbanions with known structure and charge site. In preliminary work, we have measured the gas phase basicity of these ions, and explored their chemistry with a variety of neutral reagents including $\mathrm{SO}_{2}, \mathrm{CO}_{2}, \mathrm{OCS}$, $\mathrm{CS}_{2}, \mathrm{CH}_{3} \mathrm{Cl},\left(\mathrm{CH}_{3}\right)_{3} \mathrm{CBr}$, and $\mathrm{CD}_{3} \mathrm{OD}$. A rich chemistry is observed, including proton abstraction, nucleophilic substitution, elimination, association, and hydrogen-deuterium exchange. These results are currently being prepared for publication.

Quantitative Thermochemistry. In addition to determining kinetic data for ion-molecule reactions, the FA-SIFT can provide quantitative thermochemical data, especially when combined with the results of Photoelectron Spectroscopy. As a recent example from our laboratory (Wren et al. 2012), consider 1,3,5-triazine $\left(\mathrm{C}_{3} \mathrm{H}_{3} \mathrm{~N}_{3}\right)$, an analog of benzene in which alternate carbon atoms are replaced by nitrogen. As shown in Fig. 6, the C-H bond dissociation energy $[\mathrm{BDE}(\mathrm{AH})]$ can be determined from the gas phase acidity of triazine $\left[\Delta \mathrm{H}_{\text {acid }}(\mathrm{AH})\right]$ and the electron affinity of the triazinyl radical $[\mathrm{EA}(\mathrm{A})]$, since the ionization energy of $\mathrm{H}$-atom $[\mathrm{IE}(\mathrm{H})]$ is well known. The gas phase acidity of 1,3,5-triazine was determined with the FA-SIFT by measuring the forward and reverse proton transfer rate constants with methanol, a well-characterized acid. These experiments determined $\Delta \mathrm{H}_{\text {acid }}=386.1 \pm 0.7 \mathrm{kcal} \mathrm{mol}^{-1}$. The electron affinity was measured by photodetachment studies of the triazinide anion; from energy conservation and the onset of electron detachment, it was determined $\mathrm{EA}=1.529 \pm 0.006 \mathrm{eV}$. By combining these values using the negative ion cycle in Fig. 6, the bond dissociation energy was determined to be $\mathrm{BDE}=107.8 \pm 0.7 \mathrm{kcal} \mathrm{mol}^{-1}$. This value is lower than the corresponding bond energy in benzene. This approach, which has been successfully employed by our lab for many years, can provide quantitative thermochemical data on many molecules and ions.

\section{Summary}

This presentation has provided an overview of our studies of ion chemistry of proposed DIB carriers and of ion reactions relevant to the interstellar medium. New instrumentation and novel methods of ionization allow generation and study of a wide array of ions in order to characterize their rich chemistry and quantify their thermochemistry. 


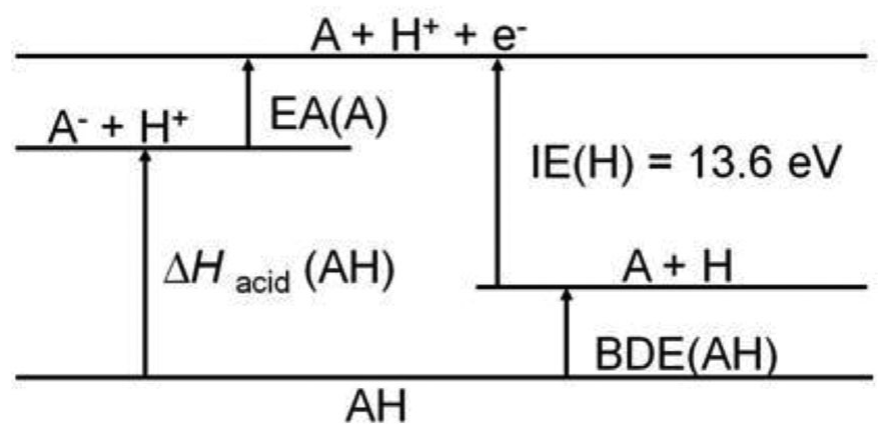

Figure 6. The Negative Ion Cycle to Determine Ion Thermochemistry.

\section{Future Directions}

Although experimental studies of gas phase ion chemistry have greatly advanced in the last decade, there remain several important challenges: (1) The study of large, nonvolatile compounds, such as extremely large PAHs, remains difficult. (2) Characterizing the site of deprotonation and/or reaction in large ions remains problematic. (3) Reactions of ions with $\mathrm{H}, \mathrm{N}$, and $\mathrm{O}$ atoms are now reasonably straightforward; however, reactions with a host of other important radical species, including $\mathrm{OH}, \mathrm{C}, \mathrm{CN}, \mathrm{CH}_{3}$, etc., are highly desirable but difficult. (4) The interstellar medium has a wide range of conditions regarding temperature and pressure; understanding ion chemistry as a function of these parameters is imperative. We welcome suggestions from the astronomy community regarding experimental studies that would provide insight into the DIBs and other important astrochemical problems.

\section{Acknowledgements}

NASA and the National Science Foundation (CHE-1012321) are gratefully acknowledged for their financial support of this research. I am grateful to Ted Snow and to all of my collaborators for their invaluable contributions to this work. I thank Nicholas Demarais for creating the Latex version of this manuscript.

\section{References}

Barckholtz, C., Snow, T. P., \& Bierbaum, V. M. 2001, ApJ, 547, L171

Demarais, N. J., Yang, Z., Martinez Jr., O., Wehres, N., Snow, T. P., \& Bierbaum, V. M. 2012, ApJ, 746, 32

Eichelberger, B., Snow, T. P., Barckholtz, C., \& Bierbaum, V. M. 2007, ApJ, 667, 1283

Fenn, J. B., Mann, M., Meng, C. K., Wong, S. F., \& Whitehouse, C. M. 1990, Mass Spectrom. Rev., 9, 37

Joblin, C. \& Tielens, A. G. G. M. (eds) 2011, PAHs and the Universe, EAS Publication Series 46 (Cambridge University Press)

Le Page, V., Snow, T. P., \& Bierbaum, V. M. 2001, ApJS, 132, 233

Shea, R. C., Habicht, S. C., Vaughn, W. E., \& Kenttämaa, H. I. 2007, Anal. Chem., 79, 2688

Snow, T. P. \& Bierbaum, V. M. 2008, Annu. Rev. Anal. Chem., 1, 229

Tulej, M., Kirkwood, D. A., Pahckov, M., \& Maier, J. P. 1998, ApJ, 506, L69

Van Doren, J. M., Barlow, S. E., DePuy, C. H., \& Bierbaum, V. M. 1987, Int. J. Mass Spectrom. Ion Proc., 81, 85

Wren, S. W., Vogelhuber, K. M., Garver, J. M., Kato, S., Sheps, L., Bierbaum, V. M., \& Lineberger, W. C. 2012, J. Am. Chem. Soc., 134, 6584

Yang, Z., Snow, T. P., \& Bierbaum, V. M. 2010, Phys. Chem. Chem. Phys., 12, 13091 
Yang, Z., Cole, C. A., Martinez Jr., O., Carpenter, M. Y., Snow, T. P., \& Bierbaum, V. M. 2011, ApJ, 739, 19

\section{Discussion}

PINO: You show experiments at room temperature; what is expected at low temperature?

BIERBAUM: Many reactions that are rapid at room temperature remain so at low temperature. However, this is not universally true. Clearly, studying ion-molecule chemistry as a function of temperature is an important challenge for future work.

FoING: Valery Le Page modeled hydrogenation and charge states by assuming constant reaction rates for the various PAHs. Is this still OK?

BIERBAUm: Yes, our recent studies indicate that hydrogenation is relatively independent of the size of the PAH cation. Therefore, Le Page's assumptions remain valid.

Pino: Why do you use Laser Induced Acoustic Desorption (LIAD) rather than Laser Desorption (LD)? How do they compare regarding stability, quantity, and softness?

BIERBAUM: Kenttämaa and her group have extensively characterized many aspects of LIAD, and her publications are a good source of detailed information. We have chosen LIAD over LD for the "softness" of the desorption process; the molecules are desorbed intact, so that we can explore the chemical reactivity of a species with known structure. We have found that the stability of the ion signal depends on the uniformity of the coating on the metal foil; in our lab, using an airbrush to deposit a solution of the PAH provides the best results and most stable signal. The quantity of material desorbed in our instrument is just adequate for carrying out experiments - we can generate mass-selected ion signal of about 5000 counts/s. The absolute quantities depend on many factors, which are discussed in the papers of Kenttämaa. 